\title{
TEORES DE FIBRA E RENDIMENTO DE GRÃOS EM POPULAÇÕES DE FEIJÃO
}

\author{
Fiber content and yield grain in common bean populations
}

\author{
Patrícia Medianeira Grigoletto Londero ${ }^{1}$, Nerinéia Dalfollo Ribeiro², Alberto Cargnelutti Filho ${ }^{3}$
}

\begin{abstract}
RESUMO
O desenvolvimento de cultivares de feijão com alto potencial de produtividade e maior teor de fibra é desejável, por isso objetivou-se neste trabalho investigar a presença de variabilidade genética para o teor de fibra e para o rendimento de grãos em populações segregantes de feijão do grupo preto. Os cruzamentos possíveis entre os genitores CNFP 8100, FT 96-1282, Varre-Sai e BRS Valente foram realizados em casa-de-vegetação. As populações obtidas $\left(F_{1}, F_{1}\right.$ recíprocos, $F_{2}$ e $\left.R C\right)$ e os genitores foram avaliados a campo durante a primavera/verão de 2003/04, em delineamento de blocos ao acaso. Apesar de ter-se utilizado genitores contrastantes para teor de fibra bruta, definidos em experimento prévio, não foram detectadas diferenças significativas para fibra bruta, fibra solúvel, fibra insolúvel, fibra alimentar total e rendimento de grãos. Assim, não foi possível obter populações segregantes com variabilidade genética, a partir dos cruzamentos entre os genitores CNFP 8100, FT 96-1282, BRS Valente e Varre-Sai. As populações obtidas apresentaram altos teores de fibra alimentar solúvel, insolúvel e total.
\end{abstract}

Termos para indexação: Phaseolus vulgaris, fibra alimentar, alimento funcional.

\section{ABSTRACT}

The development of bean cultivars with high grain yield and high dietary fiber content is desirable, thus objective of this study was to evaluate genetic variability for fiber content and grain yield in segregant populations of the black common bean. Crossings were performed inside a greenhouse among the parents CNFP 8100, FT 96-1282, Varre Sai and BRS Valente. The populations obtained $\left(F_{1}, F_{1}\right.$ reciprocals, $F_{2}$ and $\left.R C\right)$ and parents were assessed in the field during spring/summer 2003/04, using a complete randomized blocks experimental design. Significant differences were not obtained for crude fiber, soluble fiber, insoluble fiber, dietary fiber and grain yield, although contrast parents were used. In this go, it was not possible to obtain segregant populations with genetic variability of the crossing among the parents CNFP 8100, FT 96-1282, Varre Sai and BRS Valente. The populations obtained showed high soluble, insoluble and total dietary fiber content.

Index terms: Phaseolus vulgaris, dietary fiber, regular food.

(Recebido em 27 de setembro de 2006 e aprovado em 21 de junho de 2007)

\section{INTRODUÇÃO}

O feijão (Phaseolus vulgaris L.) possui componentes essenciais à dieta. As proteínas, os carboidratos, as vitaminas e os minerais, são os fatores nutricionais da maior importância (HOSFIELD, 1991). Além disso, possui conteúdo balanceado de fibra solúvel e insolúvel (HUGHES, 1991), o que é vantajoso para o consumo, em razão dos benefícios proporcionados pela ingestão da fibra.

As frações que compõem a fibra alimentar exercem efeitos fisiológicos distintos no organismo. A fração solúvel atua como importante agente hipoglicemiante e hipocolesterolemiante, ajudando no controle dos níveis de glicose no sangue, em diabéticos e na redução de risco de doenças cardiovasculares; a fibra insolúvel auxilia na redução do risco de câncer de cólon e de problemas intestinais (MOORE et al., 1998; OLSON et al., 1987; TOPPING, 1991; VANDERHOOF, 1998).

$\mathrm{O}$ teor de fibra presente nos alimentos pode ser determinado por diferentes metodologias. O método enzimático-gravimétrico considera a fibra alimentar e quantifica analiticamente os teores de fibra total e insolúvel, sendo que o teor da fibra solúvel é determinado por diferença (AOAC, 1995). Esse método é o mais indicado por utilizar enzimas no processo e, por isso, se aproxima mais do processo de digestão humana.

A determinação da composição de fibra presente nos grãos é essencial para a utilização das cultivares de feijão na composição de dietas alimentares diferenciadas. Contudo, as informações dos valores de fibra disponibilizadas

\footnotetext{
Engenheiro Agrônomo, Doutoranda - Departamento de Fitotecnia/FITO - Centro de Ciências Rurais - Universidade Federal de Santa Maria/UFSM Avenida Roraima, s/n - Camobi - 97105-900 - Santa Maria, RS - patricialondero@yahoo.com.br

${ }^{2}$ Doutora, Professora - Departamento de Fitotecnia/FITO - Centro de Ciências Rurais - Universidade Federal de Santa Maria/UFSM - Avenida Roraima, s/n - Camobi - 97105-900 - Santa Maria, RS - neiadr@smail.ufsm.br

3Doutor, Professor - Departamento de Estatística/DEST - Universidade Federal do Rio Grande do Sul/UFRGS - Avenida Bento Gonçalves, 9500, Prédio 43-111 - Agronomia - 91509-900 - Porto Alegre, RS - cargnelutti@ufrgs.br
} 
nas tabelas nacionais de composição de alimentos são determinadas, na sua grande maioria, por técnicas inapropriadas, que subestimam o valor total e não quantificam as frações solúvel e insolúvel, o que impossibilita a avaliação dos efeitos fisiológicos da fibra na dieta (OLIVEIRA et al., 1999). Entre elas, o método de Weende, que considera a fibra bruta, ainda é muito utilizado (AOAC, 1980). O valor nutricional do alimento não é quantificado adequadamente pela fibra bruta, pois na digestão ácida, parte da hemicelulose será solubilizada e, na digestão alcalina, hemicelulose e lignina serão solubilizadas, fazendo parte do extrativo não-nitrogenado, ocasionando um grave erro de estimação (SOEST, 1994).

O grão de feijão é constituído pelo tegumento, que representa cerca de $9 \%$ na matéria seca; pelos cotilédones, com 90\%; e pelo eixo embrionário, 1\% (MESQUITA, 1989). A fibra se concentra em maior quantidade no tegumento dos grãos de feijão (MENDONÇA, 2001; PAULA, 2004). Considerando que o tegumento é tecido materno, desenvolvido a partir da parede do óvulo, a geração $F_{1}$ não expressará os produtos da fertilização, pois essa geração representa o genótipo materno, a sua expressão será na geração $\mathrm{F}_{2}$ e o genótipo da geração $\mathrm{F}_{2}$ se expressará em $\mathrm{F}_{3}$ (RAMALHO et al., 2000).

Germoplasma de diferentes grupos comerciais de feijão apresentam valores diferenciados de fibra alimentar. Em feijão do grupo de cor - branco, vermelho, roxo, mulatinho e carioca, foram observados valores compreendidos entre 15,6 a 38,21\% de fibra alimentar (FA), 7,5 a $34,65 \%$ de fibra insolúvel (FI) e 1,85 a $10,2 \%$ de fibra solúvel (FS) (ACEVEDO \& BRESSANI, 1990; BECKER et al., 1986; CRUZ et al., 2004; OLIVEIRA et al., 1999). Em grãos de feijão do grupo preto foram constatados teores de 26,77 a $37,33 \%$ de FA, 22,64 a 34,22\% de FI e de 2,18 a $6,11 \%$ de FS (ACEVEDO \& BRESSANI, 1990; CRUZ et al., 2004).

A linhagem CNFP 8100 apresentou maior capacidade geral de combinação para fibra alimentar, sendo por isso recomendada para a utilização em programas de hibridação controlada, visando ao aumento do teor de fibra alimentar em feijão (LONDERO et al., 2005). Além disso, a seleção de plantas $F_{2}$ em populações segregantes desenvolvidas a partir do cruzamento BRS Valente x Varre-Sai poderá ser eficaz no desenvolvimento de germoplasma de feijão com maior teor de FA, em virtude da maior herdabilidade no sentido amplo $(97,03 \%)$ e do ganho por seleção predito $(5,36 \%$ ) (LONDERO et al., 2006a). Sendo assim, espera-se que a seleção seja eficiente para aumento do teor de fibra alimentar em feijão, pois existe variabilidade genética.
Para isso, a quantificação do teor de fibra e o conhecimento da variabilidade genética em genótipos brasileiros de feijão é necessária, uma vez que ainda são recentes as pesquisas e poucas informações disponibilizadas na literatura. Aqui objetivou-se investigar a presença de variabilidade genética para teor de fibra e para rendimento de grãos, em populações segregantes de feijão, obtidas a partir do cruzamento entre quatro genitores do grupo preto.

\section{MATERIAL E MÉTODOS}

A definição dos genitores utilizados no bloco de cruzamento foi baseada em resultados obtidos previamente (RIBEIRO et al., 2005). Assim, com base no teor de fibra bruta, selecionaram-se quatro genitores contrastantes, dois com alto teor de fibra bruta - BRS Valente $(4,97 \%)$ e VarreSai $(5,17 \%)$ - e dois com baixo teor - CNFP 8100 (3,40\%) e FT 96-1282 (3,47\%), que foram intercruzados aos pares, segundo a metodologia de dialelos completos, com a utilização dos recíprocos (CRUZ \& REGAZZI, 1997).

As hibridações controladas foram realizadas em casa-de-vegetação, em área do Departamento de Fitotecnia, da Universidade Federal de Santa Maria (UFSM), Rio Grande do Sul, utilizando a técnica sem emasculação, descrita em Peternelli \& Borém (1999). Os cruzamentos, para a obtenção das sementes $\mathrm{F}_{1}$, foram realizados durante o período de fevereiro a maio, e repetidos entre os meses de julho a novembro de 2003. As sementes $\mathrm{F}_{2}$, foram obtidas por meio da autofecundação natural das plantas $\mathrm{F}_{1}$, e as sementes dos retrocruzamentos (RC), a partir do cruzamento de planta $F_{1}$ com um dos seus genitores $\left(F_{1} \times P_{1}\right)$. A definição do genitor recorrente foi baseada no elevado teor de fibra bruta e, nas combinações em que não existia tal genitor, utilizou-se aquele de maior produtividade. Apenas para a combinação CNFP 8100 x BRS Valente, não foram obtidas sementes das gerações $\mathrm{F}_{2}$ e $\mathrm{RC}$, em razão de problemas de incompatibilidade no cruzamento.

Os blocos de cruzamentos foram conduzidos em vasos plásticos com capacidade para $5 \mathrm{~kg}$ de solo, com duas plantas por vaso. A fim de garantir o desenvolvimento normal das plantas de feijão foram realizadas irrigações diárias, controle de doenças e de insetos, sempre que necessário. À medida que as vagens de feijão atingiam a maturação fisiológica foi efetuada a colheita de forma individual.

Considerando-se que o maior teor de fibra está localizado no tegumento dos grãos de feijão, as sementes representam a expressão de uma geração anterior a que pertencem, assim, as sementes $\mathrm{F}_{2}$ representam a expressão da geração $F_{1}$, e as sementes $F_{3}$ representam a expressão 
da geração $\mathrm{F}_{2}$. Os genitores e as populações obtidas foram avaliados a campo em solo caracterizado como Alissolo Hipocrômico argilúvico típico. A semeadura ocorreu em 21 de novembro de 2003 em área do Departamento de Fitotecnia, da UFSM-RS.

O delineamento experimental utilizado foi o de blocos ao acaso, com duas repetições, e 26 tratamentos avaliados (genitores, $\mathrm{F}_{1}, \mathrm{~F}_{1}$ recíproco, $\mathrm{F}_{2}$ e $\mathrm{RC}$ ). Cada parcela foi constituída por uma linha de $1 \mathrm{~m}$ de comprimento para as populações $\mathrm{F}_{1}, \mathrm{~F}_{1}$ recíproco, $\mathrm{RC}$ e genitores, com 10 plantas por metro linear. Para as populações $F_{2}$ foram utilizadas duas linhas de $1 \mathrm{~m}$ de comprimento, por causa da presença de maior variabilidade genética.

O solo foi preparado de forma convencional e a adubação foi efetuada no sulco de semeadura, de acordo com a interpretação da análise química do solo. A adubação nitrogenada em cobertura foi parcelada em duas aplicações de $40 \mathrm{~kg} \mathrm{ha}^{-1}$ de nitrogênio nos estádios vegetativos de primeira e de terceira folhas trifolioladas, V3 e V4, respectivamente. Os tratos culturais, como controle de insetos e de plantas invasoras, foram realizados sempre que necessário, de maneira que a cultura não sofresse competição.

A colheita e a trilha das plantas foram realizadas manualmente e, após a retirada das impurezas, os grãos foram secos ao sol e em estufa $\left(65\right.$ a $\left.70^{\circ} \mathrm{C}\right)$, até atingirem umidade média de $12 \%$. Amostras de $20 \mathrm{~g}$ de grãos de cada população foram tomadas ao acaso e moídas em micro moinho até a obtenção de partículas inferiores a $1 \mathrm{~mm}$. Logo após, as amostras foram armazenadas em potes plásticos, devidamente identificados, e conservadas sob refrigeração até o momento de realização das análises de fibra bruta - segundo a metodologia descrita pela AOAC (1980), e de fibra alimentar - segundo o método adotado pela AOAC (1995).

O rendimento de grãos foi obtido em gramas por planta. As amostras para as análises foram de 20 plantas (10 plantas/repetição) para os genitores e populações $F_{1}$, $\mathrm{F}_{1}$ recíproco e retrocruzamentos. Já para as populações $\mathrm{F}_{2}$ as amostras foram de 40 plantas (20 plantas/repetição) pela maior heterogeneidade de plantas. A razão de se avaliar poucas plantas por população $\mathrm{F}_{2}$ foi por causa do alto custo das análises e do tempo demasiadamente longo requerido para a realização das mesmas, o que inviabiliza a realização do estudo em amostragens maiores.

Os resultados obtidos foram submetidos à análise da variância e as médias das populações foram comparadas entre si pelo teste de Scott-Knott, ao nível de 5\% de probabilidade.

\section{RESULTADOS E DISCUSSÃO}

Não foram observadas diferenças significativas entre os tratamentos para fibra bruta, fibra solúvel, fibra insolúvel, fibra alimentar total e rendimento de grãos, indicando a inexistência de variabilidade genética entre as populações (Tabela 1). Considerando que a interação genótipo x ambiente para teor de teor de fibra em feijão foi constatada por Londero et al. (2006b), isso explica porque os teores de fibra bruta verificados para os genitores em 2002 (RIBEIRO et al., 2005) não se repetiram no presente estudo e os genitores foram similares: BRS Valente (3,45\%), Varre-Sai (3,94\%), CNFP 8100 $(4,30 \%)$ e FT 96-1282 (3,68\%). Como a existência de variabilidade genética entre os parentais é fator primordial para a obtenção de populações com variabilidade genética, no presente estudo não foram detectadas diferenças significativas para as características avaliadas.

Os coeficientes de variação ambiental (CVe) obtidos para as características avaliadas variaram de $6,23 \%$ para fibra alimentar a 23,80\% para rendimento de grãos. Esses resultados indicam presença de efeito ambiental, principalmente para rendimento de grãos, o que poderá dificultar a seleção dessa característica associada a alto teor de fibra (Tabela 1).

O teor de fibra bruta variou de $3,42 \%$ a $4,30 \%$, enquanto que a fibra alimentar total variou de $33,39 \%$ a $39,39 \%$ (Tabela 2). Em trabalho preliminar, utilizando os genitores - BRS Valente, Varre-Sai, CNFP 8100 e FT 961282 - e as populações obtidas dos cruzamentos entre esses $-F_{1}$ e $F_{1}$ recíproco - foi possível a estratificação de diferentes grupos para fibra alimentar total (FAT) (LONDERO et al., 2005). Assim, foi proposta a seguinte classificação: alta FAT (acima de 37\%), baixa FAT (inferior a $35 \%$ ) e intermediária FAT (valores compreendidos entre as classes anteriores). Para o presente trabalho, foram obtidas as populações $\mathrm{F}_{2}$ - geração com maior variabilidade genética e heterogeneidade de plantas - e como foram incluídos os valores médios dessas gerações, e não os valores individuais de cada planta, acredita-se que esse fato tenha contribuído para aumentar o erro experimental, e como conseqüência, acrescer a diferença mínima significativa entre as médias das populações, tanto que diferenças significativas entre os tratamentos não foram observadas. Além disso, a presença do efeito materno, na expressão de um determinado caráter nos descendentes, poderá ocorrer por uma ou duas gerações (RAMALHO et al., 2000).

As diferenças de valores observados entre os teores de fibra bruta e de fibra alimentar podem ser justificadas pelos métodos de quantificação empregados. A fibra bruta é determinada pelo método de Weende que na digestão ácida, solubiliza parte da hemicelulose e na digestão 
Tabela 1 - Resumo da análise de variância para fibra bruta, fibra solúvel, fibra insolúvel e fibra alimentar total - em \% de matéria seca, e rendimento de grãos (gramas/planta), em populações de feijão. Santa Maria - RS, UFSM, 2004.

\begin{tabular}{lcccccc}
\hline \multirow{2}{*}{ Causas da Variação } & GL & \multicolumn{5}{c}{ Quadrado Médio $^{(1)}$} \\
\cline { 3 - 7 } & & Fibra Bruta & Fibra Solúvel & Fibra Insolúvel & Fibra Alimentar Total & Rendimento \\
\hline \multirow{2}{*}{ Blocos } & 1 & 0,0011 & 2,7646 & 12,6326 & 3,5517 & g/planta \\
Populações & 25 & $0,0900^{\mathrm{ns}}$ & $1,9282^{\mathrm{ns}}$ & $6,5370^{\mathrm{ns}}$ & $4,1456^{\mathrm{ns}}$ & 0,9640 \\
Resíduo & 25 & 0,0974 & 2,9559 & 6,6912 & 5,2936 & $11,1897^{\mathrm{ns}}$ \\
\hline Média & 3,81 & 8,90 & 28,02 & 36,92 & 14,6793 \\
CVe (\%) & 8,19 & 19,32 & 9,23 & 6,23 & 23,80 \\
DMS-Tukey (5\%) & 1,28 & 7,07 & 10,63 & 9,46 & 14,05 \\
\hline
\end{tabular}

(1) * = Significativo a $5 \%$ de probabilidade de erro pelo teste $\mathrm{F} ;{ }^{\mathrm{ns}}=$ não significativo.

$\mathrm{CVe}(\%)=$ coeficiente de variação ambiental.

alcalina, além da hemicelulose, a lignina também sofre extensa e variável solubilização (SOEST, 1994). De acordo com esse autor, a quantidade média de lignina, pentosanas e celulose dissolvidas na determinação de fibra bruta, para as leguminosas é de $30 \%, 63 \%$ e $28 \%$, respectivamente. Por isso, esse método é mais adequado para a avaliação de produtos destinados à alimentação animal (PICOLLI \& CIOCCA, 1999). No entanto, muitas dietas ainda são formuladas com base em informações de fibra bruta, obtidas a partir de tabelas de composição de alimentos. Por isso, apesar das limitações, o método de Weende ainda não foi totalmente descartado, pois ainda é utilizado para a composição dos cardápios dos brasileiros.

O teor de fibra solúvel variou de $8,04 \%$ a $11,11 \%$ e o teor de fibra insolúvel variou de $24,82 \%$ a $31,35 \%$, podendo-se inferir que cerca de $25 \%$ da fibra alimentar presente em grãos de feijão preto é fração solúvel (FS), enquanto que $75 \%$ é fração insolúvel (FI) (Tabela 2). Grãos de feijão branco e vermelho foram analisados pelo método de Berlin - que utiliza hidrólise com enzimas, e valores de $10,2 \%$ e $8,1 \%$ para FS e de $9,6 \%$ e $7,5 \%$ para FI foram obtidos (BECKER et al., 1986). Em feijão vermelho, foram constatados valores de 3,43\% para FS e de $12,40 \%$ para FI, após cozimento dos grãos por $40 \mathrm{~min}$ em panela de pressão, utilizando o método enzimático-gravimétrico (OLIVEIRA et al., 1999). Em amostras de feijão cozido do grupo de cor (branco, vermelho, roxo, mulatinho e carioca) e do grupo preto, foram observados valores que variaram de 1,85 a 7,06 para FS e de 15,83 a 34,65\% para FI, utilizando para análise o método enzimático-gravimétrico (CRUZ et al., 2004). Esses resultados sugerem que grãos de feijão de diferentes grupos comerciais apresentam variações para as diferentes frações da fibra alimentar.
Embora não tenha sido possível a identificação de variabilidade genética (Tabela 1), todas as populações obtidas apresentaram altos teores de fibra solúvel, insolúvel e total (Tabela 2). Considerando que a fibra solúvel apresenta efeitos hipoglicemiante e hipocolesterolemiante, o germoplasma obtido poderia ser administrado para o controle do colesterol e na alimentação de diabéticos, auxiliando no controle dos níveis de glicose no sangue e na prevenção de doenças cardiovasculares (MOORE et al., 1998; OLSON et al., 1987; TOPPING, 1991).

Além disso, a fibra insolúvel tem a função de acelerar o tempo de trânsito intestinal, auxiliando na prevenção de doenças do trato gastrointestinal (MOORE et al., 1998; OLSON et al., 1987; VANDERHOOF, 1998). Nesse sentido, o germoplasma obtido poderia ser utilizado em dietas menos calóricas e para a prevenção de constipação. Assim, as populações obtidas deverão ser melhor investigadas quanto ao seu potencial de uso na redução de risco de doenças crônicas não transmissíveis. Isso se justifica pelo fato do Instituto Nacional do Câncer recomendar um consumo diário de 20 a 30 gramas de fibra como estratégia de prevenção de câncer de cólon e de doenças do coração (BUTRUM et al., 1988).

O melhoramento concomitante das características teor de fibra alimentar e rendimento de grãos é desejado pelos melhoristas de feijão. Acredita-se que a seleção simultânea das características poderá ser efetiva em gerações mais avançadas do programa de melhoramento, desde que, depois de avaliadas as linhagens mais produtivas de feijão, seja determinado o teor de fibra alimentar nos grãos, em maior número de ambientes, em razão dos efeitos da interação genótipo x ambiente. 
Tabela 2 - Média dos teores de fibra bruta, fibra solúvel, fibra insolúvel e fibra total - em \% de matéria seca, e rendimento de grãos (gramas/planta) obtidas em populações de feijão. Santa Maria - RS, UFSM, 2004.

\begin{tabular}{|c|c|c|c|c|c|}
\hline População* & Fibra Bruta & Fibra Solúvel & Fibra Insolúvel & Fibra Total & Rendimento \\
\hline & \multicolumn{4}{|c|}{$\ldots \% \ldots$} & (g/planta) \\
\hline $\mathrm{G} 1(1 \mathrm{x} 1)$ & 4,30 & 10,00 & 27,44 & 37,44 & 10,35 \\
\hline $\mathrm{G} 2(2 \times 2)$ & 3,68 & 8,87 & 26,77 & 35,63 & 11,54 \\
\hline G3(3x3) & 3,45 & 8,57 & 24,82 & 33,39 & 13,08 \\
\hline $\mathrm{G} 4(4 \mathrm{x} 4)$ & 3,94 & 8,92 & 26,11 & 35,03 & 14,34 \\
\hline $\mathrm{F} 1 \mathrm{~A}(1 \mathrm{x} 2)$ & 3,94 & 8,04 & 31,35 & 39,39 & 11,61 \\
\hline $\mathrm{F} 1 \mathrm{~B}(1 \mathrm{x} 3)$ & 4,04 & 7,91 & 28,74 & 36,64 & 12,98 \\
\hline $\mathrm{F} 1 \mathrm{C}(1 \mathrm{x} 4)$ & 3,89 & 9,32 & 27,04 & 36,36 & 9,80 \\
\hline $\mathrm{F} 1 \mathrm{D}(2 \times 3)$ & 3,95 & 8,70 & 25,28 & 33,98 & 15,53 \\
\hline $\mathrm{F} 1 \mathrm{E}(2 \mathrm{x} 4)$ & 3,68 & 8,67 & 28,11 & 36,77 & 12,46 \\
\hline $\mathrm{F} 1 \mathrm{~F}(3 \mathrm{x} 4)$ & 3,52 & 11,11 & 26,75 & 37,86 & 18,11 \\
\hline $\mathrm{F} 1 \mathrm{~A}^{\prime}(2 \mathrm{x} 1)$ & 3,94 & 8,68 & 27,67 & 36,34 & 16,65 \\
\hline $\mathrm{F} 1 \mathrm{~B}^{\prime}(3 \mathrm{x} 1)$ & 3,86 & 7,89 & 30,46 & 38,35 & 16,92 \\
\hline $\mathrm{F} 1 \mathrm{C}^{\prime}(4 \mathrm{x} 1)$ & 3,73 & 9,44 & 28,67 & 38,11 & 13,02 \\
\hline$F 1 D^{\prime}(3 \times 2)$ & 4,01 & 10,65 & 26,63 & 37,29 & 16,61 \\
\hline$F 1 E^{\prime}(4 x 2)$ & 3,78 & 8,85 & 29,46 & 38,30 & 15,19 \\
\hline$F 1 F^{\prime}(4 x 3)$ & 3,79 & 7,00 & 30,08 & 37,08 & 17,34 \\
\hline F21(1x2)A & 4,00 & 8,67 & 27,84 & 36,50 & 12,22 \\
\hline $\mathrm{F} 22(1 \mathrm{x} 4) \mathrm{A}$ & 3,60 & 8,57 & 29,09 & 37,66 & 14,38 \\
\hline $\mathrm{F} 23(2 \times 3) \mathrm{A}$ & 4,07 & 10,81 & 25,89 & 36,69 & 13,08 \\
\hline $\mathrm{F} 24(2 \mathrm{x} 4) \mathrm{A}$ & 3,85 & 9,29 & 26,56 & 35,84 & 15,02 \\
\hline $\mathrm{F} 25(3 \mathrm{x} 4) \mathrm{A}$ & 3,73 & 8,24 & 29,77 & 38,01 & 17,55 \\
\hline $\mathrm{RC} 1(1 \times 2) \times 1$ & 3,76 & 7,75 & 28,88 & 36,63 & 18,62 \\
\hline $\mathrm{RC} 2(1 \times 4) \times 4$ & 3,42 & 9,76 & 27,12 & 36,88 & 13,15 \\
\hline $\mathrm{RC} 3(2 \times 3) \times 3$ & 3,45 & 9,23 & 27,01 & 36,25 & 14,80 \\
\hline $\mathrm{RC} 4(2 \times 4) \times 4$ & 3,82 & 8,71 & 29,38 & 38,09 & 14,49 \\
\hline $\operatorname{RC} 5(3 \times 4) \times 3$ & 3,96 & 7,77 & 31,35 & 39,39 & 14,54 \\
\hline
\end{tabular}

*:G: genitor (1. CNFP-8100; 2. FT 96-1282; 3. BRS Valente; 4. Varre-Sai).

$\mathrm{F}_{1}$ : população obtida do cruzamento entre dois genitores.

$\mathrm{F}_{2}$ : população obtida da autofecundação de plantas $\mathrm{F}_{1}$.

RC: população obtida do cruzamento entre plantas $F_{1}$ com um dos genitores.

\section{CONCLUSÕES}

A partir dos cruzamentos entre os genitores CNFP 8100, FT 96-1282, BRS Valente e Varre-Sai não foi possível obter populações segregantes com variabilidade genética para fibra e rendimento de grãos. Todas as populações obtidas apresentaram altos teores de fibra alimentar solúvel, insolúvel e total e têm potencial para uso na alimentação, para a redução de risco de doenças crônicas não transmissíveis.

\section{AGRADECIMENTOS}

Ao Conselho Nacional de Desenvolvimento Científico e Tecnológico $(\mathrm{CNPq})$ e à Coordenação de Aperfeiçoamento de Pessoal de Nível Superior (CAPES), pelas bolsas concedidas. 


\section{REFERÊNCIAS BIBLIOGRÁFICAS}

ACEVEDO, E.; BRESSANI, R. Contenido de fibra dietetica y digestibilidad del nitrogeno en alimentos centroamericanos: Guatemala. Archivos Latinoamericanos de Nutrición, Caracas, v. 40, n. 3, p. 439-451, 1990.

ASSOCIATION OF OFFICIAL AGRICULTURAL CHEMISTS. Official methods of analysis. 13. ed. Washington, DC, 1980. 2000 p.

ASSOCIATION OF OFFICIAL AGRICULTURAL CHEMISTS. Official methods of analysis. 16. ed. Washington, DC, 1995. $2000 \mathrm{p}$

BECKER, H. G; STELLER, W.; FELDHEIN, W.; WISKER, E.; KULIKOWSKI, W.; SUCKOW, P.; MEUSER, F.; SEIBEL, W. Dietary fiber and bread: intake, enrichment, determination and influence on colonic function. Cereal Foods World, Saint Paul, v. 31, n. 4, p. 306-310, Apr. 1986.

BUTRUM, R. R.; CLIFFORD, C. K.; LANZA, E. NCI dietary guidelines: rationale. American Journal of Clinical Nutritional, New York, v. 48, n. 3, p. 881-895, Sept. 1988.

CRUZ, C. D.; REGAZZI, A. J. Modelos biométricos aplicados ao melhoramento genético. 2. ed. Viçosa: UFV, 1997. $390 \mathrm{p}$.

CRUZ, G. A. D. R.; OLIVEIRA, M. G. A.; PIRES, C. V.; PILON, A. M.; CRUZ, R. S.; BRUMANO, M. H. N.; MOREIRA, M. A. Avaliação da digestibilidade protéica, inibidor de protease e fibras alimentares de cultivares de feijão (Phaseolus vulgaris L.). Brazilian Journal of Food Technology, Campinas, v. 7, n. 2, p. 103-109, jul./ dez. 2004.

HOSFIELD, G. L. Genetic control of production and food quality factors in dry bean. Food Technology, Chicago, v. 45, n. 9, p. 98-103, Sept. 1991.

HUGHES, J. S. Potential contribution of dry bean dietary fiber to health. Food Technology, Chicago, v. 45, n. 9, p. 122-126, Sept. 1991.

LONDERO, P. M. G.; RIBEIRO, N. D.; RODRIGUES, J. A.; POERSCH, N. L.; TRENTIN, M. Genetic variability for dietary fiber content in common bean populations. Crop Breeding and Applied Biotechnology, Viçosa, v. 5, n. 1, p. 86-90, Mar. 2005.
LONDERO, P. M. G.; RIBEIRO, N. D.; CARGNELUTTI FILHO, A.; RODRIGUES, J. A.; ANTUNES, I. F. Herança dos teores de fibra alimentar e rendimento de grãos em populações de feijoeiro. Pesquisa Agropecuária Brasileira, Brasília, v. 41, n. 1, p. 51-58, jan. 2006a.

LONDERO, P. M. G.; RIBEIRO, N. D.; POERSCH, N. L.; ROSA, S. S. da; JOST, E.; CARGNELUTTI FILHO, A.; ANTUNES, I. F.; NÖRNBERG, J. L. Variabilidade genética de cultivares de feijão para fibra alimentar. In: REUNIÃO SUL BRASILEIRA DE FEIJÃO, 8., 2006, Londrina. Anais... Londrina: IAPAR, 2006b. p. 96-98.

MENDONÇA, C. V. C. E. Caracterização química e enzimática de famílias de feijões obtidas do cruzamento das linhagens amarelinho e CI-107. 2001. 48 p. Dissertação (Mestrado em Agroquímica e Agrobioquímica) - Universidade Federal de Lavras, Lavras, 2001.

MESQUITA, I. A. Efeito materno na determinação do tamanho da semente do feijoeiro (Phaseolus vulgaris L.). 1989. 70 f. Dissertação (Mestrado em Genética e Melhoramento de Plantas) - Universidade Federal de Lavras, Lavras, 1989.

MOORE, M. A.; PARK, C. B.; TSUDA, H. Soluble and insoluble fiber influences on cancer development. Critical Reviews in Oncology/Hematology, New York, v. 27, n. 3, p. 229-242, 1998.

OLIVEIRA, L. F. A.; OLIVEIRA, M. G. A.; COSTA, N. M. B.; COELHO, D. T. Teores de fibra alimentar e de inibidores de proteases em arroz polido (Oriza sativa, L.) e feijão comum (Phaseolus vulgaris, L.). Brazilian Journal of Food Technology, Campinas, v. 2, n. 1/2, p. 161-165, 1999.

OLSON, A.; GRAY, M. G.; CHIU, M. C. Chemistry and analysis of soluble dietary fiber. Food Technology, Chicago, v. 4, n. 2, p. 71-82, Feb. 1987.

PAULA, S. R. R. Efeito materno associado à capacidade de cozimento do feijoeiro. 2004. 53 p. Dissertação (Mestrado em Melhoramento Genético Vegetal) - Universidade Federal de Lavras, Lavras, 2004.

PETERNELLI, L. A.; BORÉM, A. Hibridação em feijão. In: BORÉM, A. Hibridação artificial de plantas. Viçosa: UFV, 1999. p. 269-294 
PICOLLI, L.; CIOCCA, M. L. S. Métodos para avaliação de fibra alimentar. In: RIBEIRO, A. M. L.; BERNARDI, M. L.; KESSLER, A. M. Tópicos em produção animal. Porto Alegre: UFRGS, 1999. p. 307-322.

RAMALHO, M. A. P.; SANTOS, J. B.; PINTO, C. A. B. P. Genética na agropecuária. Lavras: UFLA, 2000. 472 p.

RIBEIRO, N. D.; LONDERO, P. M. G.; HOFFMANN JUNIOR, L.; POERSCH, N. L.; CARGNELUTTI FILHO, A. Dissimilaridade genética para proteína e fibra em grãos de feijão dos grupos preto e de cor. Revista Brasileira de
Agrociência, Pelotas, v. 11, n. 2, p. 167-173, abr./jun. 2005.

SOEST, P. J. van. Nutritional ecology of the ruminant. 2. ed. Ithaka: Cornell University, 1994. 476 p.

TOPPING, D. L. Soluble fiber polysaccharides: effects on plasma cholesterol and colonic fermentation. Nutrition Reviews, Catalunya, v. 49, n. 7, p. 195-203, July 1991.

VANDERHOOF, J. A. Immunonutrition: the role of carbohydrates. Nutrition, Philadelphia, v. 14, n. 7/8, p. 595598, July/Aug. 1998. 